\title{
REVIEW OF GRANAT OBSERVATIONS OF AGNS
}

\author{
E.CHURAZOV, M.GILFANOV, A.FINOGUENOV, R.SUNYAEV, \\ M.CHERNYAKOVA, YU.APALKOV, S.GREBENEV and I.LAGUNOV \\ Space Research Institute, Profsoyuznaya 84/32, 117810 Moscow, Russia
}

E.JOURDAIN, J.P.ROQUES, P.MANDROU and L.BOUCHET

Centre d'Etude Spatiale des Rayonnements, 9, avenu du Colonel Roche, BP 4946, 91029

Toulouse Cedex, France

and

\author{
J.BALLET, A.CLARET, P.LAURENT and F.LEBRUN \\ Service d'Astrophysique, Centre d'Etudes Nucleaires de Saclay, Orme des Merisiers, 91191 \\ Gif-sur-Yvette Cedex, France
}

\begin{abstract}
Brief review of AGNs observations in the X-ray / soft gamma-ray bands with the orbital observatory GRANAT is presented.

For three well known bright objects (3C273, NGC4151 and Cen A) broad band (3 keV few hundreds $\mathrm{keV}$ ) spectra have been obtained. Imaging capabilities allowed accurate (several arcminutes) identification of these objects with sources of hard $\mathrm{X}$-rays.

The spectrum of NGC4151 above $\approx 50 \mathrm{keV}$ was found to be much steeper than that in most of the previous observations, while in standard X-ray band the spectrum agrees with observed previously. The comparison of the observed spectra with that of the X-Ray Background (XRB) indicates that sources similar to NGC4151 could reproduce the shape of XRB spectrum in 3-60 $\mathrm{keV}$ band.

Cen A was observed in the very low state during most of observations in 1990-1993, except for two observations in 1991. The variability of the hard X-ray flux has been detected on the time scales of several days.
\end{abstract}

Key words: X-Rays: AGNs

\section{Introduction}

Two coded aperture imaging X-ray telescopes ART-P and SIGMA on board the GRANAT satellite have been operated since 1989 December 1. ART-P telescope consists of four identical modules, having $5^{\prime}$ angular resolution over $3^{\circ} .6 \times 3^{\circ} .6$ fully coded field of view, and operates in standard (3-30 keV) energy range (Sunyaev et al., 1990). The SIGMA telescope, operating in hard X-ray / Soft gamma-ray band, provides $11^{\circ} .4 \times 10^{\circ} .6$ images (at half-sensitivity) with nominal angular resolution of $\sim 15^{\prime}$ (Paul et al., 1991). More than $40 \approx$ one-day observations of 3C273, NGC4151 and Cen $A$ have been performed. These objects were unambiguously identified with sources of hard X-rays with several arcminutes accuracy (except for possible contamination of $3 \mathrm{C} 273 \mathrm{flux}$ due to GRS1227+02/1E1227+0224). Shown in Fig. 1 are the $4^{\circ} \times 4^{\circ}$ SIGMA images in $35-150 \mathrm{keV}$ energy range averaged over most of observations of these three objects in 1990-1993.

The results of AGNs observations with GRANAT during first years of the mission are presented in a number of papers by Apalkov et al., 1992 (ART-P, observations in 1990, 3C273, NGC 4151, Cen A); Ballet et al., 1991, 1992a,b (SIGMA

63

T. J.-L. Courvoisier and A. Blecha: Multi-Wavelength Continuum Emission of AGN, 63-72.

(C) 1994 IAU. Printed in the Netherlands. 

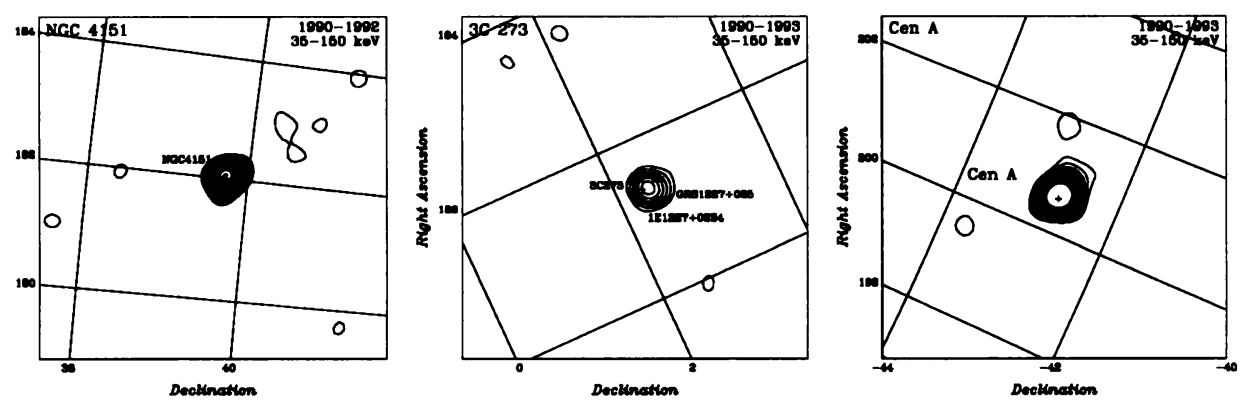

Fig. 1. Slices of SIGMA images in 35-150 keV band, averaged over most of observations in 1990-1993 of NGC4151 (a), 3C273 (b) and Cen A (c). Contours are 3,4,..,15o levels. The images have been convolved with the detector Point Spread Function in order to increase the sensitivity to the point sources. As the result apparent angular resolution (width of the peaks on the images) is considerably worse than the best possible resolution.

and ART-P, 1990-1992, 3C273, NGC 4151, Cen A); Jourdain et al., 1992 (SIGMA, 1990, NGC4151); Bassani et al., 1992 (SIGMA, 1990, 3C273); Jourdain et al., 1993 (SIGMA, 1990-1991, Cen A); Lebrun et al., 1992 (SIGMA, 1990-1991, M87 and NGC4388); Bassani et al., 1993 (SIGMA, 1992, 3C273, NGC4151, Cen A), Finoguenov et al., 1993 (ART-P \& SIGMA, 1990-1992, NGC4151). We will concentrate below on the data of observations in the hard X-ray / soft Gamma-ray band obtained by GRANAT/SIGMA.

\section{NGC 4151}

NGC 4151 is one of the nearest $\left(d \sim 20 \mathrm{Mpc}\right.$ for $\left.H_{0}=50 \mathrm{~km} \mathrm{~s}^{-1} \mathrm{Mpc}^{-1}\right)$ lowluminosity Seyfert galaxies. It has 2-10 keV photon index of $\alpha \sim 1.5 \pm 0.2$ (Yaqoob et al., 1993) somewhat harder than "canonical" value of $\alpha \sim 1.7$ for Sy I galaxies. In standard X-ray band the flux varies by at least factor of 5 with the fastest variability detected so far of the order of $1 / 2$ day. Observations at higher energies detected power law spectrum with indices of $\approx 1.7$ up to hundreds of $\mathrm{keV}$ or even $\mathrm{MeV}$ (see Perotti et al., 1991 for review) placing NGC 4151 among the hardest objects on the sky in this energy range.

The source has been observed by SIGMA 19 times in 1990-1992 (see Table 1 for the list of sessions with highest quality of the data). In contrast to previous high-energy observations the spectrum observed during first 6 sessions in 1990 was found to be very steep with photon index $\alpha \sim 3.1 \pm 1$ in the 30-200 keV energy band (Jourdain et al., 1992). On the other hand the observations of ART-P on 1990 November $11,12,16,19$ and 20 gave the value of the $3-20 \mathrm{keV}$ index of 
TABLE I

The list of SIGMA/GRANAT observations of NGC 4151

\begin{tabular}{|c|c|c|}
\hline Ses. \# & Date (UT) & Exposure, (hours) \\
\hline 0122 & $12.579-13.47307 / 90$ & 17.78 \\
\hline 0123 & $13.636-13.88007 / 90$ & 5.00 \\
\hline 0190 & $15.576-16.301 \quad 11 / 90$ & 14.40 \\
\hline 0191 & $16.526-17.218 \quad 11 / 90$ & 13.83 \\
\hline 0193 & $19.664-20.593 \quad 11 / 90$ & 18.39 \\
\hline 0194 & $20.831-22.193 \quad 11 / 90$ & 27.01 \\
\hline 0304 & $29.457-30.52306 / 91$ & 21.01 \\
\hline 0306 & $11.425-12.47107 / 91$ & 20.75 \\
\hline 0367 & $19.470-20.29711 / 91$ & 16.38 \\
\hline 0368 & $20.455-21.86911 / 91$ & 28.01 \\
\hline 0470 & 24.648-25.547 06/92 & 17.99 \\
\hline 0531 & $18.492-19.255 \quad 11 / 92$ & 15.08 \\
\hline 0532 & $19.377-20.230 \quad 11 / 92$ & 17.00 \\
\hline 0534 & 23.377-24.229 $11 / 92$ & 17.00 \\
\hline
\end{tabular}

$\alpha=1.48 \pm 0.03$ (Apalkov et al., 1992), i.e. typical for this source at these energies. All further SIGMA observations confirmed the steepness of NGC 4151 spectrum at high energies (Finoguenov et al., 1993). Observations of OSSE in 1991 (Maisack et al., 1993) also show similar very steep spectrum at energies above $65 \mathrm{keV}$, suggesting that steep state is now the dominant state for NGC 4151.

The spectrum of NGC 4151 averaged over all useful SIGMA observations in 1990-1992 is shown in Fig. 2. The assumption of power law spectrum in 35-300 $\mathrm{keV}$ range with photon index less than 2 can be rejected at the confidence of $99 \%$. (Finoguenov et al., 1993). Comparison of the SIGMA spectrum with the ART-P data (Apalkov et al., 1992) suggests the break somewhere between 30 and $100 \mathrm{keV}$ (Fig. 2). The position of the break in the broken power law approximation was estimated to be $\sim 50 \mathrm{keV}$ (best fit to the 3-300 keV GRANAT data in November 1990; Finoguenov et al., 1993). The similar value can be inferred from Fig. 2, but since shown ART-P and SIGMA spectra are not contemporaneous one should treat this result with caution.

The SIGMA data (in 35-200 keV energy range) can be satisfactory approximated by the power law with index $\alpha=2.3 \pm 0.2$ indicating the steepening from standard to hard X-rays of at least $\Delta \alpha \sim 0.8$ (see Table 4) The SIGMA data are consistent with further steepening of the spectrum $(\alpha \approx 3.4)$ above $\sim 100$ $\mathrm{keV}$ found by OSSE/CGRO (Maisack et al., 1993). So broad band spectrum over few $\mathrm{keV}$ - few hundred keV range (ART-P,SIGMA, OSSE) requires at least two breaks in broken power law approximation (Finoguenov et al., 1993). The broad band spectrum of NGC4151 can be satisfactory approximated by the models in- 




Fig. 2. The Spectra of NGC 4151, 3C273 and Cen A averaged over ART-P observations in 1990 and SIGMA observations in 1990-1993. The points below $1 \sigma$ have been replaced with $1 \sigma$ upper limits.

volving the exponential cutoff at high energies. Such a behavior, when the spectrum rapidly steepens with energy, argues in favor of the thermal models of origin of the $\mathrm{X}$-ray spectra (see also Zdziarski et al., 1993).

The absorption corrected $2-10 \mathrm{keV}$ flux $(20 \pm 1) \times 10^{-11} \mathrm{ergs}^{-2} \mathrm{sm}^{-1}$ measured by ART-P in November 1990 is considerably higher than $\sim 11 \times 10^{-11} \mathrm{ergs} \mathrm{cm}^{-2} \mathrm{~s}^{-1}$ expected for $\alpha=1.48$ from index/luminosity relation obtained in the frame of nonthermal models considered by Yaqoob \& Warwick (1991). On the other hand rather similar combinations of photon index and 2-10 keV flux have been observed by Ginga in 1991 January (c.f. Jan 91d from Table 3 of Yaqoob \& Warwick ,1993).

Variability of the X-ray flux has been detected both in standard (Apalkov et al., 1992) and hard X-ray bands (Finoguenov et al., 1993). Shown in Fig. 3 is the 35-150 keV light curve of NGC4151 in 1990-1992 obtained by GRANAT. The probability (according to $\chi^{2}$ test) that measurements correspond to constant source flux is about $3 \%$.

The AGNs are known to contribute at least $30-40 \%$ to the X-Ray Background (hereafter XRB) at $2 \mathrm{keV}$. The shape of XRB spectrum in board 3-60 keV band can be well approximated by the emission of optically thin plasma with temperature $\approx 45 \mathrm{keV}$ (Rothschild et al., 1983). Below $30 \mathrm{keV}$ it has power law slope of $\approx$ 


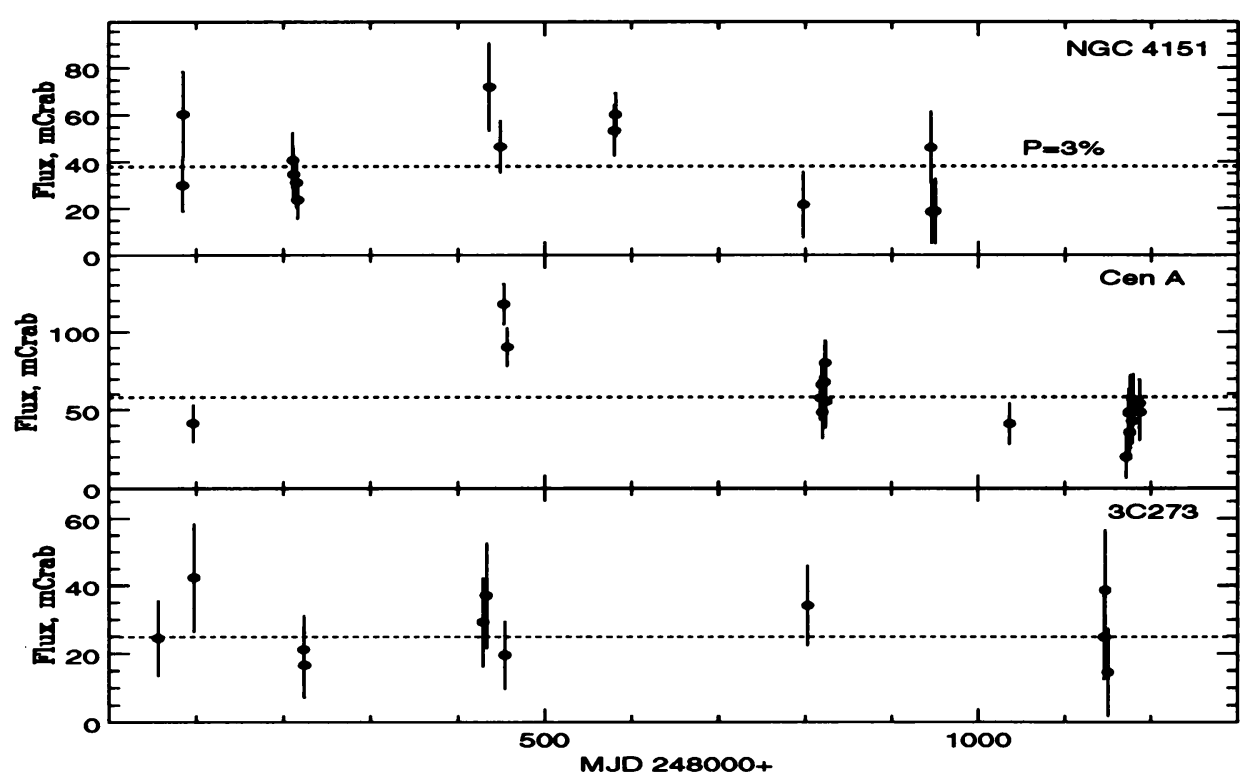

Fig. 3. The light curves of NGC4151, Cen $A$ and $3 C 273$ in 35-150 keV band according to SIGMA measurements in 1990-1993

1.4 (photon index) and steepens to index $\approx 2.4$ in $30-200 \mathrm{keV}$ range. However there is obvious lack of bright objects having the spectra of the similar shape. The assumption that dominant contribution in this energy band come from the objects with "canonical" power law spectra (photon index $\approx 1.7$ ) extending to hundreds $\mathrm{keV}$ is in conflict (1987) with XRB shape - the latter is flatter at low energies and steeper at high.

Although the NGC4151 is probably rather unique source it is interesting that the spectrum of this object observed by GRANAT well satisfy the requirements mentioned above. Indeed NGC4151 has almost proper slope at low energies and steepens at high. Shown in Fig. 4 is the expected shape of background due to the objects with spectra similar to that of NGC4151 in comparison with measured spectrum of XRB. Two curves (with and without evolution of luminosities are shown for illustration). The fit to the spectrum of NGC4151 observed by GRANAT in 1990 November (Finoguenov et al., 1993) has been adopted $\left(I_{\nu}=E^{-\alpha} * \exp (-E / k T), \alpha=0.32, k T=79 \mathrm{keV}, N_{H}=5 \times 10^{22} \mathrm{~cm}^{-2}\right)$. 


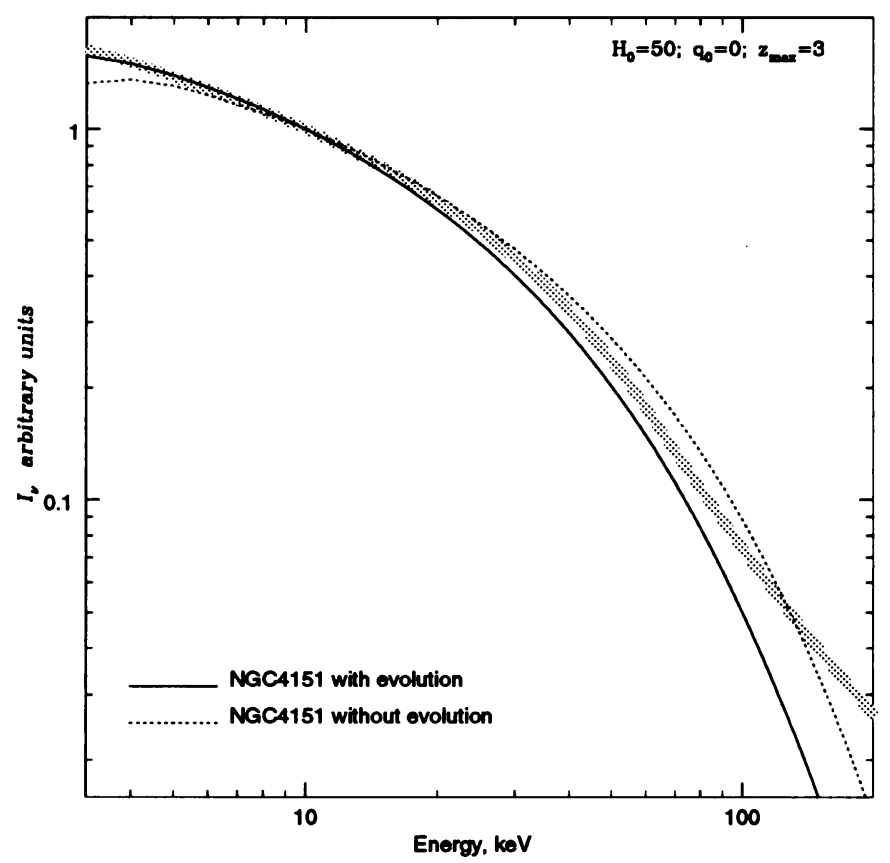

Fig. 4. The expected shape of the diffuse background due to the sources with spectra similar to that of NGC4151, observed by GRANAT. The following parameters were adopted: $z_{\max }=3$; $q_{o}=0 ; H_{0}=50$. Two thin curves correspond to the case when all objects have the same luminosity and when luminosity evolves according to the model proposed by Maccacaro et al., 1991. Thick line is the empirical approximation of XRB spectrum (Gruber, 1992).

\section{3C 273}

Numerous observations of this nearby $(z=0.158)$ QSO have detected the power law-spectrum extending to hundreds $\mathrm{keV}$ with nearly the same photon index $\alpha=1.4$.

The list of SIGMA observations of $3 \mathrm{C} 273$ with highest quality of data are given in Table 2. In the first observations the source was found in the state similar to that observed previously (Bassani et al., 1992). Shown in Fig. 2 is the spectrum of 3C273 in broad energy band observed with GRANAT. The ART-P data points have been averaged over the observations on 1990 (Apalkov et al., 1992); the SIGMA points have been averaged over all observations in 1990-1992. Unlike NGC 4151 there is no significant evidence for strong break of the spectrum from standard to hard X-ray bands. The photon index in 3-24 keV range $(\alpha=1.4 \pm 0.05)$ (Apalkov et al., 1992) 
TABLE II

The list of SIGMA/GRANAT observations of 3C273

\begin{tabular}{llc}
\hline Ses.\# & Date (UT) & Exposure,(hours) \\
\hline 0104 & $14.431-15.58906 / 90$ & 23.00 \\
0130 & $25.467-26.11807 / 90$ & 13.01 \\
0197 & $27.875-28.59311 / 90$ & 14.22 \\
0198 & $28.805-29.70811 / 90$ & 18.00 \\
0300 & $21.742-22.55306 / 91$ & 16.00 \\
0302 & $25.752-26.14806 / 91$ & 8.01 \\
0309 & $16.670-17.50807 / 91$ & 16.64 \\
0474 & $29.612-30.36306 / 92$ & 15.00 \\
0610 & $7.427-8.46306 / 93$ & 20.62 \\
0611 & $8.595-9.04106 / 93$ & 9.00 \\
0613 & $11.511-12.47006 / 93$ & 19.00 \\
0614 & $12.598-13.34606 / 93$ & 11.50 \\
\hline
\end{tabular}

is consistent with inferred from $35-200 \mathrm{keV}$ SIGMA data $(\alpha=1.3 \pm 0.32)$. However the source is rather weak in SIGMA energy band and substantially steeper slopes in $35-200 \mathrm{keV}$ band $(\alpha \approx 1.6-1.7)$ can not be ruled out with SIGMA data.

Although ART-P and SIGMA observations are not simultaneous there is no strong mismatch of spectra at $30 \mathrm{keV}$. The whole set of SIGMA data points in $35-150 \mathrm{keV}$ band is consistent with the assumption of constant flux. The marginal evidence for variability based on first few SIGMA measurements (see Fig. 3) has been mentioned by Bassani et al., 1992. The average flux at $100 \mathrm{keV}$ of $(1.86 \pm$ $0.21) \times 10^{-5} \mathrm{ph} / \mathrm{cm}^{2} / \mathrm{s} / \mathrm{keV}$ found by SIGMA is in general compatible with previous measurements.

Another source GRS1227+025 has been reported 15' apart from 3C273 (Jourdain et al., 1992). It was relatively bright only during short period of time (observations 197 and 198 in Table 2). Grindlay (1992) recently pointed out that Einstein HRI source 1E1227+0224 (Tananbaum et al., 1979) is located within the $5^{\prime}$ SIGMA error circle reported for GRS1227+025. Note that image of this field shown in Fig. 1 has been convolved with detector Point-Spread-Function which cause apparent broadening of the peaks. For detailed discussion see Jourdain et al., 1992; Grindlay, 1992.

\section{Cen A}

Cen $\mathrm{A}$ is radiogalaxy best studied in $\mathrm{X}$-rays. This object has rather low luminosity $\left(L_{3-10 \mathrm{keV}} \approx 10^{42} \mathrm{ergs} / \mathrm{s}\right)$, however due to small distance to the source $(\sim 5 \mathrm{Mpc})$ Cen $A$ is the brightest extragalactic source seen by GRANAT.

Cen $\mathrm{A}$ is known to be strongly variable both in standard and hard X-ray band. 
TABLE III

The list of SIGMA/GRANAT observations of Cen A

\begin{tabular}{lccc}
\hline Ses.\# & Date (UT) & Exposure,(hours) \\
\hline 0129 & $24.388-25.261$ & $07 / 90$ & 17.26 \\
0308 & $15.381-16.471$ & $07 / 91$ & 21.42 \\
0311 & $19.507-20.596$ & $07 / 91$ & 21.57 \\
0482 & $14.529-15.443$ & $07 / 92$ & 18.04 \\
0483 & $15.574-16.422$ & $07 / 92$ & 16.91 \\
0484 & $16.553-17.048$ & $07 / 92$ & 10.00 \\
0485 & $18.475-19.464$ & $07 / 92$ & 19.59 \\
0486 & $19.587-20.42307 / 92$ & 16.61 \\
0487 & $20.545-21.14207 / 92$ & 12.00 \\
0567 & $17.656-18.556$ & $02 / 93$ & 18.00 \\
0625 & $2.515-3.365$ & $07 / 93$ & 17.00 \\
0626 & $5.463-6.379$ & $07 / 93$ & 18.17 \\
0627 & $6.511-7.337$ & $07 / 93$ & 16.44 \\
0628 & $7.457-7.750$ & $07 / 93$ & 6.00 \\
0629 & $9.615-10.525$ & $07 / 93$ & 18.03 \\
0630 & $10.645-11.33707 / 93$ & 13.88 \\
0635 & $17.696-18.46107 / 93$ & 15.18 \\
0636 & $18.581-19.22907 / 93$ & 13.00 \\
\hline
\end{tabular}

The variability of $35-150 \mathrm{keV}$ flux is also apparent from SIGMA data (Fig. 3). In the most of observations the source was in very low state in hard X-rays comparable only with the HEXE/MIR observations in June 1987 (Maisack et al., 1991). The hard X-ray flux from Cen A seems to gradually decrease in 1992 to 1993. The Cen A was found in the relatively high state only during two observations in 1991 (observations 308 and 311 in Table 3). These two observations were separated by 4 days and the flux in the latter observation was significantly lower than in the first one (Jourdain et al.,1993). If the fast decrease of the flux between these two observations characterizes also the rise time, than it is possible that the source remained in the low state during whole period of SIGMA observations in 19901993 and two observations in 1991 July occasionally happened during relatively short flare of the source. Observations of ART-P in 1990 (Apalkov et al., 1992) in standard X-ray band also revealed the source in relatively low state in comparison with most of previous measurements (Morini et al., 1989).

According to preliminary analysis there is evidence for spectral variation in SIGMA data between the states with high and low flux at $100 \mathrm{keV}$ (observations 308, 311 in 1991 and all observations in 1992 and 1993 respectively). The power law photon index in $35-200 \mathrm{keV}$ is $\approx 1.76$ for 1991 observations (flux at $100 \mathrm{keV}$ $\left.F_{100} \sim 6.7 \times 10^{-5} \mathrm{phot} / \mathrm{s} / \mathrm{cm}^{2} / \mathrm{keV}\right)$ and $\approx 2.3$ for the spectrum averaged over 
TABLE IV

The power law approximations of NGC4151, 3C273 and Cen A spectra, averaged over observations of ART-P (1990) and SIGMA (1990-1993)

\begin{tabular}{llllll}
\hline Source & $N_{H}^{a}$ & Flux $1 \mathrm{keV}^{b}$ & $3-24 \mathrm{keV}$ index & Flux @ $100 \mathrm{keV}^{c}$ & $35-200 \mathrm{keV}$ index \\
\hline NGC4151 & $6 \pm 2$ & $3.6 \pm 0.2$ & $1.48 \pm 0.03$ & $2.06 \pm 0.21$ & $2.3 \pm 0.2$ \\
Cen A & $9 \pm 2$ & $9.6 \pm 0.5$ & $1.69 \pm 0.02$ & $4.16 \pm 0.23$ & $1.98 \pm 0.11$ \\
3 C 273 & $0.018^{d}$ & $1.2 \pm 0.1$ & $1.40 \pm 0.05$ & $1.86 \pm 0.21$ & $1.3 \pm 0.3$ \\
\hline
\end{tabular}

Errors are $68 \%$ confidence limits for single parameter estimates

a - in units of $10^{22} \mathrm{~cm}^{-2}$

b $-10^{-2} \mathrm{ph} / \mathrm{cm}^{2} / \mathrm{s} / \mathrm{keV}$

c $-10^{-5} \mathrm{ph} / \mathrm{cm}^{2} / \mathrm{s} / \mathrm{keV}$

${ }^{d}-$ fixed

1992-1993 observations $\left(F_{100} \sim 3.0 \times 10^{-5} \mathrm{phot} / \mathrm{s} / \mathrm{cm}^{2} / \mathrm{keV}\right)$. The spectral shape in 1992-1993 observations is probably more complicate than simple power law over $35-200 \mathrm{keV}$ band - it is somewhat steeper below $\approx 50 \mathrm{keV}$ and somewhat flatter in 50-150 keV bands. The analysis of the observations in 1992 and 1993 is in progress now and detailed results will be published elsewhere.

The spectrum of Cen A averaged over ART-P observations in 1990 (Apalkov et al., 1992) and SIGMA in 1990-1993 is shown in Fig. 2. In standard X-ray band the source had photon index $\approx 1.7$ while in SIGMA $35-200 \mathrm{keV}$ band it is $\approx 1.98$ (see Table 4). There is evidence for steepening of the spectrum above $\approx 150 \mathrm{keV}$ in agreement with results of OSSE/CGRO (Johnson et al., 1993).

\section{References}

Apalkov Yu. et al., 1992, in the Proc. 28th Yamada Conf. on Frontiers of X-Ray Astronomy, ed. Y.Tanaka, K.Koyama, p.251

Ballet J. et al., 1991, 26th Moriond conference on "The early observable universe from diffuse backgrounds", Rocca-Volmerange, Deharveng and Tran Thanh Van editors, Editions Frontieres, 43

Ballet J. et al., 1992a, Heidelberg conference on "Physics of Active Galactic Nuclei", ed. Duschl and Wagner, Springer Verlag, 67

Ballet J. et al., 1992b, Garching conference on "X-ray emission from Active Galactic Nuclei and the X-ray background", ed. Brinkmann and Truemper, MPE report 235, p 50

Bassani L. et al., 1992, ApJ, 396, 504

Boldt E., 1987, Phys. Rep., 146, 215

Finoguenov A.et al., 1993, in preparation

Grindlay J., 1992, A\&AS , 97, 113

Gruber D.E., 1992, In The X-ray background, eds. X.Barcons \& A.C.Fabian, Camb. Univ. Press.

Johnson W.N. et al., 1993, A\&AS , 97, 21

Jourdain E. et al., 1992, A\&A, 256, L38

Jourdain E. et al., 1993, A\&A, 412, 586

Lebrun F. et al., 1992, A\&A, 264, 22

Maccacaro T. et al., 1991, ApJ, 374, 117 
Maisack M. et al., 1989, 23rd ESALB Symp., "X-Ray Astronomy", eds. J.Hunt \& B.Battrick, 975.

Maisack et al., 1993, ApJ, 407, L67

Morini M., Anselmo F. \& Molteni D., 1989, ApJ, 347, 750

Paul J. et al., 1991, Adv.Space Res., 11, (8) 289

Perotti F. et al., 1991, ApJ, 373, 75

Rothschild R.E. et al., 1983, ApJ, 269, 423

Sunyaev R. et al., 1990, Adv.Space Res., 10, (2) 233

Tananbaum H. et al., 1979, ApJ, 234, L9

Yaqoob T. \& Warwick R.S., 1991, MNRAS, 248, 773

Yaqoob T. et al., 1993, MNRAS, 262, 435.

Zdziarski A. et al., 1993, ApJ, 414, L93 